\title{
SUPERPLASTICITY AND JOINING OF ZIRCONIA-BASED CERAMICS
}

A. Dominguez-Rodriguez, F. Gutierrez-Mora, and M. Jimenez-Melendo. Dpto. de Física de la Materia Condensada, Universidad de Sevilla, 41080 Sevilla, Spain.

R. Chaim.

Dept. of Materials Engineering, Technion, Israel Institute of Technology, Haifa 32000 , Israel.

J. L. Routbort

Energy Technology Division, Argonne National Laboratory.

November 1999

The submitted manuscript has been created by the
University of Chicago as Operator of Argonne
National Laboratory ("Argonne") under Contract
No. W-31-109-ENG-38 with the U.S. Department of
Energy. The U.S. Government retains for itself, and
others acting on its behalf, a paid-up, nonexclusive,
irrevocable worldwide license in said article to
reproduce, prepare derivative works, distribute
copies to the public, and perform publicly and
display publicly, by or on behalf of the Government.

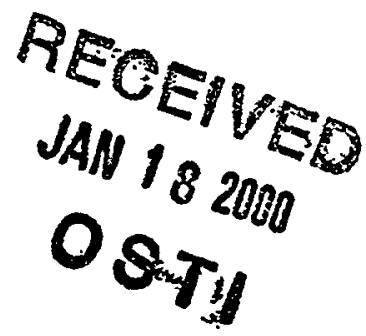

This research was supported by CICYT under grant no MAT97-1007-C02 (Ministerio de Educación y Ciencia-Spain), by the Fund for the promotion of Research at the Technion (1998) and by the Israel Ministry of Science. J. Routbort is grateful to IBERDROLA for providing funding his visit to the Universidad de Sevilla and acknowledges the support of the U.S. Department of Energy under Contract W-31-109-Eng-38.

Manuscript submitted to Materials Research Society for annual meeting, Boston, MA November $29^{\text {th }}-$ December $2^{\text {und }}, 1999$ 


\section{DISCLAIMER}

This report was prepared as an account of work sponsored by an agency of the United States Government. Neither the United States Government nor any agency thereof, nor any of their employees, make any warranty, express or implied, or assumes any legal liability or responsibility for the accuracy, completeness, or usefulness of any information, apparatus, product, or process disclosed, or represents that its use would not infringe privately owned rights. Reference herein to any specific commercial product, process, or service by trade name, trademark, manufacturer, or otherwise does not necessarily constitute or imply its endorsement, recommendation, or favoring by the United States Government or any agency thereof. The views and opinions of authors expressed herein do not necessarily state or reflect those of the United States Government or any agency thereof. 


\section{DISCLAIMER}

Portions of this document may be illegible in electronic image products. Images are produced from the best available original document. 


\title{
SUPERPLASTICITY AND JOINING OF ZIRCONIA-BASED CERAMICS
}

F. Gutierrez-Mora, A. Dominguez-Rodriguez and M. Jimenez-Melendo.

Dpto. de Física de la Materia Condensada, Universidad de Sevilla, 41080 Sevilla, Spain.

R. Chaim. and G. B. Ravi.

Dept. of Materials Engineering, Technion, Israel Institute of Technology, Haifa 32000, Israel.

\author{
J. L. Routbort \\ Energy Technology Division, Argonne National Laboratory.
}

\begin{abstract}
Steady-state creep and joining of alumina/zirconia composites containing alumina volume fractions of 20,60 , and $85 \%$ have been investigated between 1250 and $1350^{\circ} \mathrm{C}$. Superplasticity of these compounds is controlled by grain-boundary sliding and the creep rate is a function of alumina volume fraction, not grain size. Using the principles of superplasticity, pieces of the composite have been joined by applying the stress required to achieve 5 to $10 \%$ strain to form a strong interface at temperatures as low as $1200^{\circ} \mathrm{C}$
\end{abstract}

\section{Introduction}

The high-temperature plastic deformation of alumina ceramics has been extensively reported in the literature [1-3]. Achieving high ductility in pure alumina is difficult because of rapid static and dynamic grain growth that is accompanied by large strain hardening and severe cavitation. The relatively high grain-boundary energy (GBE) in pure alumina [1] provides a high driving force for grain growth. The high GBE is also responsible for the relatively low cohesive strength that results in severe cavitation [1].

Suppression of grain growth can be achieved through low temperature sintering or/and the use of additives. Although the solubility of a second phase in alumina is very limited, the use of additives such as $\mathrm{MgO}, \mathrm{ZrO}_{2}$ or $\mathrm{Y}_{2} \mathrm{O}_{3}$ inhibit the grain growth by segregation of these cations to the alumina grain boundaries $[1,4]$.

In the case of alumina/zirconia, microstructure and mechanical properties has been widely studied $[1,4-7]$ and it has been shown that the equiaxed fine microstructure is preserved during deformation improving the ductility of this compounds $[1,6,7]$.

In this paper we will characterize the mechanism controlling the plasticity as a function of the alumina volume fraction and of the phase distribution.

It is well known that grain boundary sliding (GBS) controls the macroscopic deformation of these compounds [6]. When two pieces of ceramics are compressed in the superplastic regime, the grains in the interface rotated with respect to each other producing interpenetration and a strong junction of both pieces [8-10]. Therefore, superplastic flow has been used to join alumina/zirconia ceramics and the conditions and strength of the junctions will be also discussed in this paper.

\section{Experimental conditions}

Alumina/zirconia composites with alumina volume fractions of 20,60 and $85 \%$, and with different phase distribution, were investigated. The compositions are designated as ZT20A, ZT60A and ZT85A. Fabrication processes of the ZT60A and ZT85A were described in detail in references 11 and 12 , respectively.

The ZT20A was fabricated as following: nanocrystalline $\mathrm{ZrO}_{2}-4$ wt\% $\mathrm{Y}_{2} \mathrm{O}_{3}$ (Y-TZP) powder with mean particle size of $10 \mathrm{~nm}$ was prepared by chemical precipitation from the 
nitrate salt solutions, as previously described [13]. The commercial nanocrystalline alumina powder (Neomat) was composed of transition alumina ( $\theta$ and $\partial$ ) with a mean particle size of $10 \mathrm{~nm}$. The Y-TZP and alumina powders were separately dispersed ultrasonically in ethanol; the $\mathrm{pH}$ of the dispersions were adjusted to 1.5 and 7 respectively. The two slurries were ball milled for $24 \mathrm{~h}$ before mixing; the resultant $\mathrm{pH}$ was between 1.5 and 2 . At this stage two different mixing and sedimentation routes were adopted. In the case of the sedimentation (S designated samples), ammonia solution was added to the final mixed slurry to change its $\mathrm{pH}$ to 10.5. Then the slurry was left to sediment overnight; the latter was collected and washed with ethanol and dried at $200^{\circ} \mathrm{C}$ for 1 day. In the case of the milled ( $\mathrm{M}$ designated samples), the mixed slurry was dried using a heating plate with continuous mixing to avoid sedimentation. Once the slurry was dried, the wet powder was dried at $200^{\circ} \mathrm{C}$ for 1 day. Cylindrical pellets of $15 \mathrm{~mm}$ in diameter and length were uniaxially cold pressed followed by cold isostatic pressing at $200 \mathrm{MPa}$. Finally, the pellets were sintered at $1600^{\circ} \mathrm{C}$ for $2 \mathrm{~min}$ using heating and cooling rates of $5{ }^{\circ} \mathrm{C} / \mathrm{min}$ and $20^{\circ} \mathrm{C} / \mathrm{min}$, respectively.

The average grain size of zirconia and alumina were determined by electron microscopy and the final density was determined by the Archimedes method. The phase content was determined by $\mathrm{X}$-ray diffraction.

The samples were cut into parallelepiped shape having dimensions of $6 \times 3 \times 3 \mathrm{~mm}$ and compressed in air at temperatures between 1200 and $1350^{\circ} \mathrm{C}$ at constant load (creep experiments) or at constant cross-head speed in a Instron machine. In these latter experiments a steady-state stress, as defined by a zero work-hardening rate, was obtained after $1-2 \%$ of deformation. The stress of the plateau is the flow stress for Instron experiments. The stress ranged between 5 and $500 \mathrm{MPa}$ and the initial strain rate varied between $10^{-7}$ and $10^{-4} \mathrm{~s}^{-1}$.

For the junction, pieces of ZT20AM and ZT60A were compressed in an Instron machine to a strain of 5 and $10 \%$ at crosshead speed of $5 \mu \mathrm{m} / \mathrm{min}$ that corresponded to an initial strain rate, $\dot{\varepsilon} \sim 10^{-5} \mathrm{~s}^{-1}$, for the dimensions of the pieces joined. The temperatures employed were between 1200 and $1300^{\circ} \mathrm{C}$.

The junction strength was measured from cracks produced by a $100 \mathrm{~N}$ Vickers indentor whose diagonal was along the interface.

\section{Experimental results and discussion}

The ZT20AS specimens were characterized by a more homogeneous distribution of

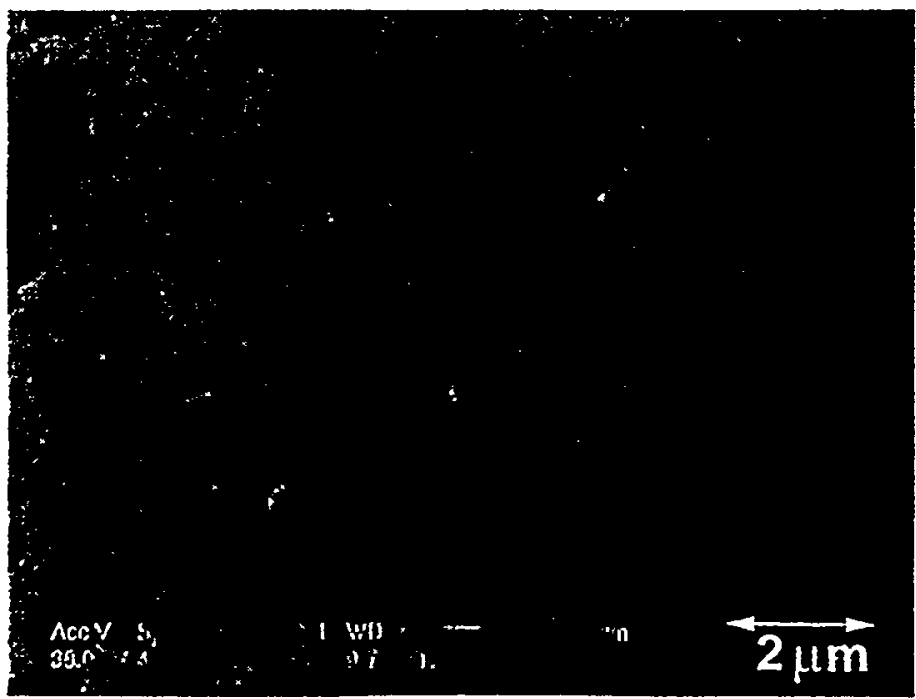

Fig 1. Microstructure of the as-received ZT20AS 
both alumina and zirconia grains compared with the ZT20AM specimens. Fig. 1 is a typical micrograph of the microstructure of a ZT20AS ceramics. Scanning electron microscopy (SEM) $[11,12]$ revealed that the ZT60A and ZT85A consisted of a very homogeneous distribution of both phases. The characteristics of these ceramics are listed in Table I. No differences in the grain size of both phases were found in the two types of ZT20A.

The superplastic behavior of alumina/zirconia composites has been analyzed using the equation:

$$
\dot{\varepsilon}=A \sigma^{n} d^{-p} \exp \left(\frac{-Q}{R T}\right)
$$

where $\dot{\varepsilon}$ is the strain rate, $\sigma$ is the applied stress, $d$ the grain size, $R$ and $T$ have their usual meaning, and $\mathrm{n}, \mathrm{p}$ and $\mathrm{Q}$ are the creep parameters characterizing the mechanism controlling the creep behavior.

TABLE I. Grain size of both phases of the materials used in this work.

\begin{tabular}{|c|c|c|}
\hline Material & $\mathrm{Al}_{2} \mathrm{O}_{3}$ grains $(\mu \mathrm{m})$ & $3 \mathrm{Y}$ grains $(\mu \mathrm{m})$ \\
\hline ZT20A & 0.8 & 0.6 \\
\hline ZT60A & 0.7 & 0.4 \\
\hline ZT85A & 1.3 & 0.5 \\
\hline
\end{tabular}

Figs. $2 \mathrm{a}$ and $2 \mathrm{~b}$ are creep curves typical of all materials tested. From stress and temperature changes it is possible to determine $n$ and $Q$ as shown. There are no

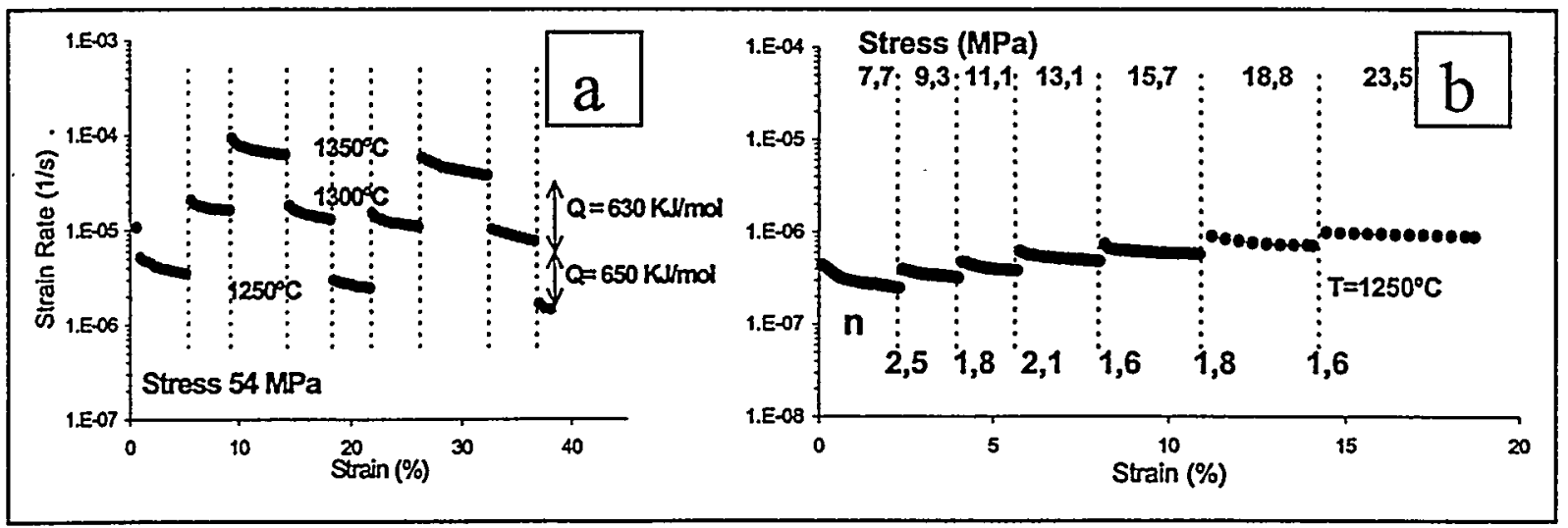

Fig 2. Typical creep curves to determine the activation energy $Q(a)$ and the parameter $n$ (b).

discontinuities in the extrapolation of the creep data for a given temperature, despite the fact that $T$ was changed several times. Therefore, the creep data indicate that the microstructure remained constant during the test without grain growth. No grain-size exponent was determined because the grain size was not varied.

The effect of $T$ and $\sigma$ is shown in Fig. 3, which is a plot of $\log \varepsilon$ vs $\log \sigma$ for three different temperatures. The excellent agreement between the creep and Instron data is further evidence that microstructure was unchanged and that the tests were performed in steady state. That is, the final state is path independent. The slopes of these curves are close to 2 decreasing at the higher stresses. $\mathrm{Q}$ and $\mathrm{n}$ were determined from curves as in fig. 2. Values of $\mathrm{n}$ at low and intermediate stresses of $1.9 \pm 0.1$ and of $1.4 \pm 0.1$ at the higher stresses were measured. The values of $\mathrm{n}$ and $\sigma$ are independent of the composition and phase distribution. Fig. 4 shows that there is no influence of the phase distribution. As shown in fig. 4 , the value of 2 at stresses as low as $5 \mathrm{MPa}$ indicates that the threshold stress is zero in these compounds. The same behavior has been reported in Y-TZP with low purity $[14,15]$. 
A $\mathrm{n}$ value of 2 has been also measured by Wakai et al. [6] for stresses below $80 \mathrm{MPa}$ and by Clarisse et al [7]. However, these last authors found than $\mathrm{n}$ tends to 1 when the stress increases.

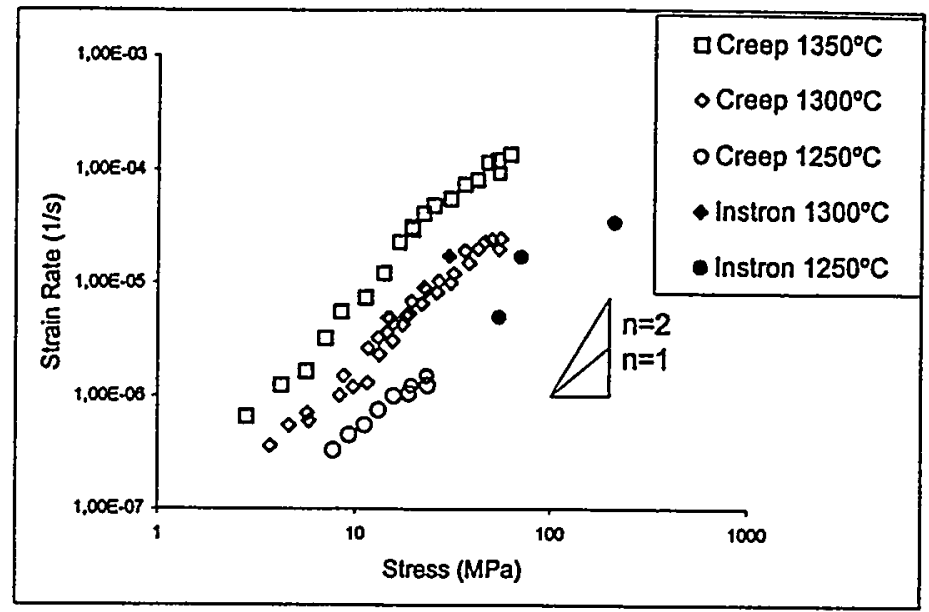

Fig. 3: Plot of $\log \varepsilon$ versus $\log \sigma$ for different temperatures.

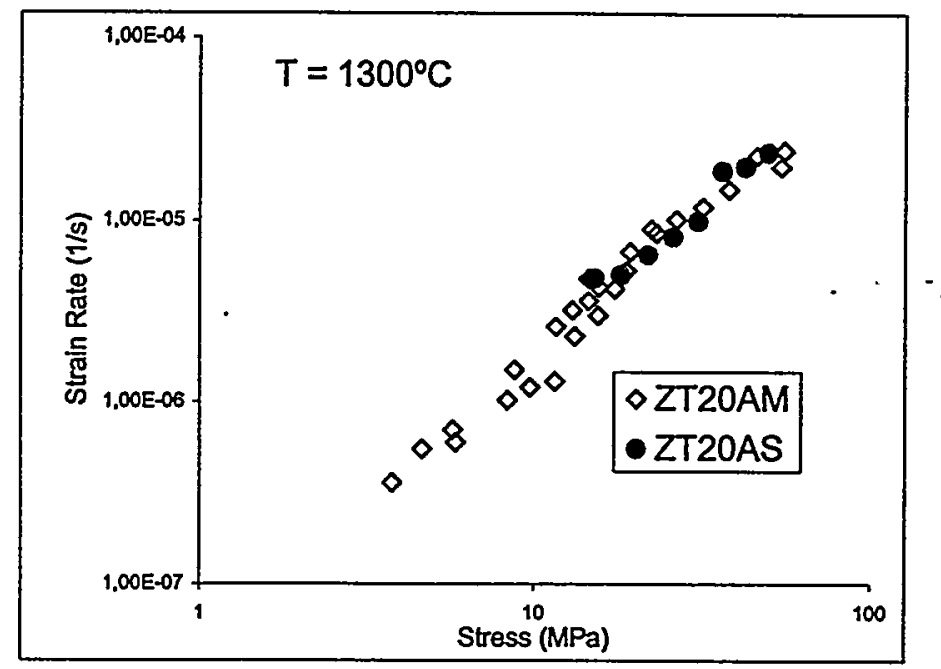

Fig.4: Influence of phase distribution in the mechanical behavior.

The $Q$ value was weakly dependent on stress. A Q-value of $680 \pm 20 \mathrm{~kJ} / \mathrm{mol}$ at the lower stresses and of $640 \pm 10 \mathrm{~kJ} / \mathrm{mol}$ for stresses higher than $50 \mathrm{MPa}$ were measured. Although the activation energy for non-doped alumina [7] and pure Y-TZP [14] ranged between $450-500 \mathrm{~kJ} / \mathrm{mol}$, a higher value of $Q$ has been systematically found for the different alumina/zirconia composites $[6,7,16]$. The higher $\mathrm{Q}$ has been attributed to the interdiffusion of $\mathrm{Zr}$ to the $\mathrm{Al}_{2} \mathrm{O}_{3}$ phase resulting in the modification of the defect formation enthalpy [7].

As already mentioned, the suppression of grain growth in alumina can be achieved through the use of additives as zirconia. The zirconium, in alumina, precipitates to the interface $[1,6,7]$ lowering the GBE and improving the stability of the microstructure and the superplastic formability of these compounds. At the same time, the segregation of $\mathrm{Zr}^{4+}$ at the grain boundaries reduces the aluminum diffusivity. For instance, the $\mathrm{Al}^{3+}$ in these compounds changes its diffusivity with respect to pure alumina, increasing the activation energy from 500 $\mathrm{kJ} / \mathrm{mol}$ in pure alumina to $700 \mathrm{~kJ} / \mathrm{mol}$ in alumina/zirconia composites $[5,6]$. No variation in $\mathrm{Q}$ with the zirconia/alumina composition has been detected. This result is compatible with stability of the microstructure of these compounds when the second phase is present at 
relatively low volume fractions $[1,7]$, as a consequence of the precipitation of zirconium at the grain boundaries and the change of the diffusivity.

In table II the creep resistant for the three compositions used in this work are presented together with the results in the literature, for a stress of $50 \mathrm{MPa}$ and two different temperatures of 1300 and $1350^{\circ} \mathrm{C}$. It is obvious that the mechanical behavior of these compounds depend on the alumina volume fraction and not of the grain size. For instance, in our work, ZT20A and ZT60A have almost the same grain size, however the strain rate is one order of magnitude lower for ZT60A.

TABLE II. Strain Rates at a stress of $50 \mathrm{MPa}$ for different compositions and two temperatures.

\begin{tabular}{|c|c|c|c|c|c|}
\hline Material & Authors & $\begin{array}{c}\mathrm{Al}_{2} \mathrm{O}_{3} \text { grains } \\
(\mu \mathrm{m})\end{array}$ & $\begin{array}{c}3 \mathrm{Y} \text { grains } \\
(\mu \mathrm{m})\end{array}$ & $\begin{array}{c}\text { Strain Rate } \\
(1 / \mathrm{s}) 1300^{\circ} \mathrm{C}\end{array}$ & $\begin{array}{c}\text { Strain Rate } \\
(1 / \mathrm{s}) 1350^{\circ} \mathrm{C}\end{array}$ \\
\hline ZT20A & Present work & 0.8 & 0.6 & $3 \times 10^{-5}$ & $1 \times 10^{-4}$ \\
ZT20A & {$[7]$} & 0.6 & 0.6 & $4 \times 10^{-5}$ & $2 \times 10^{-4}$ \\
ZT50A & {$[7]$} & 1.1 & 0.8 & $6 \times 10^{-6}$ & $3 \times 10^{-5}$ \\
ZT50A & {$[6]$} & 0.6 & 0.5 & $6 \times 10^{-6}$ & $2.5 \times 10^{-5}$ \\
ZT60A & Present work & 0.7 & 0.4 & $3 \times 10^{-6}$ & - \\
ZT69A & {$[6]$} & 1.0 & 0.6 & $1 \times 10^{-6}$ & $7 \times 10^{-6}$ \\
ZT80A & {$[7]$} & 1.4 & 0.7 & - & $7 \times 10^{-6}$ \\
ZT85A & Present work & 1.3 & 0.5 & Broken & $1 \times 10^{-6}$ \\
ZT86A & {$[6]$} & 1.0 & 0.5 & $.1 \times 10^{-6}$ & $7 \times 10^{-6}$ \\
\hline
\end{tabular}

After deformation, the microstructure remained unchanged (not shown here) with the same grain size and shape as measured by the form factor. These characteristics are typical when GBS is the main mechanism controlling plasticity.

Based on the GBS mechanism, two types of junction have been produced. In one case, two pieces of TZ20A were joined and in the other case one piece of TZ20A was joined with another of TZ60A. Fig. 5 shows the interface of the junction. The interface has almost

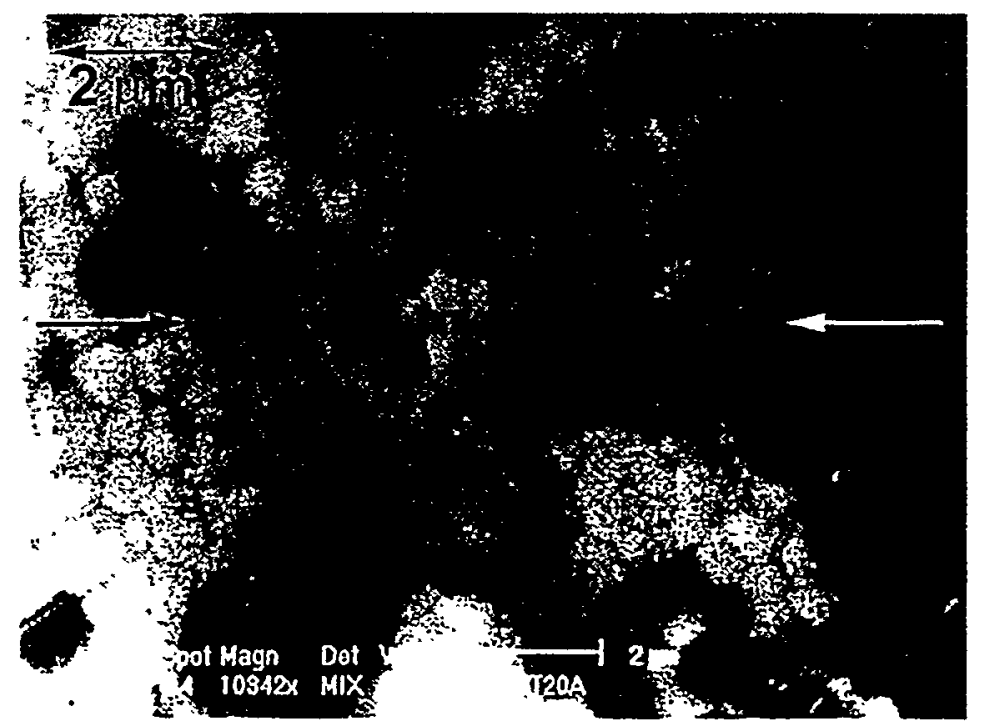

Fig. S: Junction of two pieces of ZT20A. The arrows indicate the location of the interface.

disappeared, showing that the grains of one part interpenetrate the other. The junction is formed by rotation of the grains of both pieces. The crack lengths of the Vickers indentations developed along and perpendicular to the interface in the case of ZT20A/ZT20A are 
$280 \pm 30 \mu \mathrm{m}$ and $270 \pm 20 \mu \mathrm{m}$. In the case of ZT20AVTT60A, the cracks did not propagate along the interface but deviate into the brittle material (ZT60A). These results indicate that the pieces were perfectly joined.

\section{Conclusion}

It is shown that the ZTA composites due to the refinement of the microstructure of the alumina and to the presence of YTZP became more damage tolerant that pure alumina although the strength is controlled by this phase.

These compounds can be deformed superplastically at temperatures as low as $1250^{\circ} \mathrm{C}$. The main mechanism of the plastic deformation at high temperature of these compounds is GBS accommodated by diffusion process, which are controlled at their turn by the precipitation of $\mathrm{Zr}$ at the grain boundaries.

Based on the GBS mechanism, different pieces of ZTA has been joined at temperatures as low as $1200^{\circ} \mathrm{C}$ for period of time as long as $30 \mathrm{~min}$. The cracks develop along the interface after Vickers indentation show that the pieces were perfectly joined.

\section{Acknowledgement:}

This research was supported by CICYT under grant no MAT97-1007-C02 (Ministerio de Educación y Ciencia-Spain), by the Fund for the promotion of Research at the Technion (1999) and by the Israel Ministry of Science. J. Routbort is grateful to IBERDROLA for providing funding for his visit to the Universidad de Sevilla.

\section{References}

1. I.W. Chen and L. A. Xue J. Am. Ceram. Soc., 73, p. 2585 (1990)

2. W. R. Cannon in Advances in Ceramics, Vol 10 Structure and Properties of MgO and $\mathrm{Al}_{2} \mathrm{O}_{3}$ Ceramics. Edited by W.D. Kingery. Am. Ceram. Soc. Columbus OH. 1984. p. 741-49. 3. W. R. Cannon and T. G. Langdon, J. Mater. Sci, 18, p. 1 (1983). Ibidem, 23, p. 1 (1998). 4. T. Sakuma, Y. Ikuhara, Y. Takigawa and P. Thavorniti, Mat. Sci. Eng. A234-236, p. 226 (1997).

5. H. Yoshida, K. Okada, Y. Ikuhara and T. Sakuma. Phil. Mag. Let. 76, p. 9 (1997).

6. F. Wakai, T. Nagano and T. Iga. J. Am. Ceram. Soc. 80, p. 2361 (1997)

7. L. Clarisse, R. Baddi, A. Bataille, J. Crampon, R. Duclos and J. Vincens. Acta Mater. 45, p. 3843 (1997).

8. J. Ye and A. Dominguez-Rodriguez, Scripta Met. and Mater. 33, p. 441 (1995).

9. A. Dominguez-Rodriguez, F. Guiberteau and M. Jimenez-Melendo. J. Mat. Res. 13, p. 1631 (1998).

10. A. Dominguez-Rodriguez, E. Jimenez-Pique and M. Jimenez-Melendo. Scripta Mater. 39, p. 21, (1998).

11. M. Jimenez-Melendo, C. Clauss, A. Dominguez-Rodriguez, G. De Portu, E. Roncari and P. Pinasco, Acta Mater. 46, p. 3995 (1998).

12. M. Jimenez-Melendo, C. Clauss, A. Dominguez-Rodriguez, A. J. Sanchez-Herencia, and J. S. Moya, J. Am. Ceram. Soc. 80, p. 2126 (1997).

13. G. Hare'l, B. G. Ravi, and R. Chaim, Mater. Lett. 39, p. 63 (1999).

14. M. Jimenez-Melendo, A. Dominguez-Rodriguez and A. Bravo-Leon. J. Am. Ceram. Soc. 81, p. 2761 (1998)

15. A. H. Chokshi, Mat. Sci. Eng. A166, p.119 (1993).

16. M. Jimenez-Melendo and A. Dominguez-Rodriguez, Phil. Mag. A, 79, p.1591 (1999). 